\title{
Voyage aux pays des fleurs : pensée politique et conte merveilleux dans l'œuvre de George Sand
}

\author{
Pascale Auraix-Jonchière \\ Université Clermont Auvergne, CELIS \\ pascale.auraix.jonchiere@neuf.fr
}

Rebut: 15 de gener de 2015

Acceptat: 20 de maig de 2015

RESUM

Viatge al país de les flors: pensament polític i conte meravellós en l'obra de George Sand

Aquest conte de George Sand explica les aventures d'un noi, confrontat a un univers meravellós i ple d'animals. Però el seus viatges condueixen també el personatge cap a diferents regions literàries: es desplaça de gènere en gènere, de manera que el conte de fades pren un valor d'apòleg. El viatge hi té, doncs, un valor metafòric i condueix el lector a un ric espai intertextual en què textos $\mathrm{i}$ imatges voregen.

Paraules Clau

Viatge, conte, faula, apòleg, gèneres literaris, intertextualitat.

RÉSUMÉ

Voyage au pays des fleurs : pensée politique et conte merveilleux dans l'œuvre de George Sand

Ce conte de George Sand raconte les aventures d'un jeune garçon, confronté à un univers merveilleux et animalier. Mais ses voyages conduisent aussi le personnage dans diverses contrées littéraires : il se déplace de genre en genre, de sorte que le conte de fées prend une valeur d'apologue. Le voyage y prend donc une valeur métaphorique et conduit le lecteur dans un riche espace intertextuel où se côtoient textes et images.

Mots Clés

Voyage, conte, fable, apologue, genres littéraires, intertextualité. 


\section{RESUMEN \\ Viaje al país de las flores: pensamiento político y cuento maravilloso en la obra de George Sand}

Este cuento de George Sand explica las aventuras de un joven, confrontado a un universo maravilloso y lleno de animales. Pero sus viajes conducen también al personaje hacia diferentes regiones literarias: se desplaza de género a género, de manera que el cuento de hadas adquiere un valor de apólogo. El viaje posee en este cuento un valor metafórico y conduce al lector a un rico espacio intertextual en el que se codean textos e imágenes.

Palabras Clave

Viaje, cuento, fábula, apólogo, géneros literarios, intertextualidad.

\section{AbSTRACT}

Travelling to the «flower land »: political thought and fairy tale in George Sand's work

George Sand's tale relates the adventures of a young boy who has to cope with different animals while travelling in a wonderful land. But this character crosses various literary countries as well : he moves from a literary genre to another, so that finally the fairy tale converts into an allegory. Travel is then a metaphor and guides the reader into a rich intertextual space in which texts and pictures coexist.

KeYWORDS

Travel, fairy tale, allegory, literary genres, intertextuality.

En février 1844, un texte intitulé Les Fleurs de mai paraît dans l'Almanach du mois. Texte insolite, il reste difficile à déterminer à cause de sa forme inclassable : il s'agit d'une adresse à diverses fleurs printanières. Sa structure repose sur la reprise d'apostrophes (« Jacinthe blanche au cœur vert », «Cyclamen de la Brenta », «Bruyère blanche »...) qui relancent l'énoncé et constituent comme autant de notations mineures encadrées par un syntagme majeur : "Belles fleurs de mai », délicate expansion du syntagme titre. La présence d'interjections lyriques, la dimension visionnaire des scènes décrites, le déploiement du titre en refrain, concourent à créer un indéniable effet 
poétique. Or ce « cantique floral $^{1} »$ a paradoxalement une finalité idéologique : il s'agit d'opposer un passé révolu, fait d'inconscience et de repli sur soi, à un présent désespérément lucide où le « je » se détourne de son amour ancien pour les fleurs impuissantes et s'inquiète du sort de son prochain ${ }^{2}$. Le locuteur, qui s'adresse donc aux fleurs insouciantes, accuse : "Vous ne savez pas ce que souffrent les hommes », "vous n'aimez pas, vous ne sentez pas, vous ne connaissez pas » (p. 137). La sensibilité poétique associée au monde floral se met au service d'une démonstration à la portée tout à la fois idéologique et éthique qui repose sur une nécessaire mise à distance.

Dès lors, le monde des fleurs est séparé de celui des hommes, pris à partie dans une relation d'interlocution qui se caractérise dans un premier temps par l'emploi de la négation et de l'antithèse. Ainsi, s'adressant à la jacinthe : « tes sœurs fleurissent loin de moi, et je n'ai rien à leur demander qu'elles puissent me donner [...] ; l'homme a besoin des autres hommes ${ }^{3} »$. Les fleurs constituent un univers à part entière, retranché ; il reste étranger aux préoccupations des hommes confrontés au Mal : "Vous n'êtes que de vains fantômes de l'immortelle beauté ${ }^{4}$ ». Toutefois le traitement du motif floral évolue tout au long de l'article : si la souffrance et la haine excluent de recourir à ces « froids emblèmes de l'impérissable harmonie ${ }^{5}$ », la construction d'un monde idéal et égalitaire par « les enfants déshérités de la civilisation, les mendiants et les parias, troupeau du Christ », à l'écart de la civilisation corrompue, «sur les sommets nus et chauves des sommets incultes ${ }^{6} »$, pourrait avoir pour corollaire une floraison nouvelle. Dans ce contexte original, le re-fleurir signe une réinvention des codes, désormais indissociable d'un déplacement (des hommes) et d'une transplantation (des fleurs) : « vous irez dans la demeure des hommes réconciliés vous marier aux naïves fleurs de la solitude $^{7} »$. C'est pourquoi la conclusion du texte assigne aux fleurs nouvelles un mode de signification inédit :

\footnotetext{
${ }^{1}$ Pour reprendre l'expression de François KERLOUEGAN dans son article « Motif floral et défaillances de l'éros dans Lélia » dans Fleurs et jardins dans l'œuvre de George Sand, Simone BERNARD-GRIFFITHS et Marie-Cécile LEVET (dir.), Clermont-Ferrand, PUBP, 2006, coll. « Révolutions et Romantismes », n¹0, p. 369.

2 « Belles fleurs de mai, orgueil et jeunesse de la terre, je ne vous aime plus, vous que j'ai tant aimées ! », in George SAND, Nouvelles (1831-1853, Éditions Paléo, 2011, p. 137.

${ }^{3}$ Ibid., p. 136. Je souligne.

${ }^{4}$ Ibid., p. 137.

${ }^{5}$ Ibid.

${ }^{6}$ Ibid., p. 138.

${ }^{7}$ Ibid., p. 139.
} 
Oh ! alors, riantes conquêtes de la civilisation nouvelle, symboles de la poésie ressuscitée, palmes aux mains de l'esclave affranchi, couronnes au front de la Liberté, je vous rendrai mon culte et mes soins, ô belles fleurs que j'ai tant aimées ${ }^{8}$ !

Le texte s'achève ainsi sur la vision utopique d'un monde qui réhabilite les parias mis au ban de la société, en relation avec un renouvellement des codes symboliques. Le mouvement de la pensée repose sur un passage de l'exclusion à la fusion, qui se traduit par un déplacement tout à la fois spatial et sémantique. Or cette configuration - penser une nouvelle genèse, qui associerait la création d'une société utopique à celle d'un monde idéal, celui des fleurs - servira de fondement à plusieurs récits à venir, où le monde floral s'identifie à une contrée singulière. C'est en particulier le cas d'un conte, Histoire du véritable Gribouille, écrit en 1850 à l'intention de la jeune Valentine Fleury ${ }^{9}$, et publié l'année suivante chez Blanchard, illustré de vignettes par Maurice Sand.

\section{Un conte dynamique : variations sur le voyage}

Le protagoniste de ce « petit conte ${ }^{10} »$ en deux parties, le jeune Gribouille, se présente sous la figure d'un voyageur paradoxal. Dans un premier temps en effet les trajets répétés qui le conduisent de son foyer — où des parents cupides et mal aimants et une fratrie persécutrice le harcèlent - au château de M. Bourdon, riche seigneur des environs, lui sont imposés.

C'est par intérêt que ses parents l'envoient au château, espérant quelque largesse de la part de celui qui semble avoir élu l'enfant et désirer " faire sa fortune » $(H G$, p. 21). Les aller-retours entre ces deux lieux structurent la première partie du conte. Voyageur malgré lui, Gribouille suit les ordres qui organisent sa destinée, contre sa volonté propre : «Quand Gribouille fut sur le chemin du château que sa mère lui avait indiqué, il se sentit bien fatigué » ( $\mathrm{p}$. 22) ; « Or donc, au bout de deux ou trois jours, on l'habilla misérablement, on lui mit une veste toute déchirée [...] et on l'envoya ainsi chez M. Bourdon » (p. 38) ; « Le jour suivant, on mit sur un âne un beau baril de miel superbe, et on

\footnotetext{
${ }^{8} \mathrm{Ibid}$.

${ }^{9}$ Valentine Fleury, 1838-1931, fille d'Alphonse Fleury, originaire de la Châtre qui fut élu représentant du peuple en 1848. Valentine a douze ans en 1850.

${ }^{10}$ George SAND, Histoire du véritable Gribouille, Paris, Blanchard, 1851, dédicace. Les références renverront à cette édition et seront données entre parenthèses, sous l'abréviation $H G$.
} 
envoya Gribouille chez M. Bourdon. » (p. 42). Le quatrième de ces voyages imposés prend une allure définitive :

Petit, lui dit [son père], retournez de ce pas chez M. Bourdon. Dites-lui que votre père vous donne à lui, et gardez-vous d'en marquer le moindre déplaisir. Restez avec lui, je vous le commande ( $H G$, p. 46)

Si le terme de « voyage » peut s'appliquer à ces déplacements, c'est en raison de leur côté aventureux et incertain, de leur longueur relative aussi, puisque Gribouille souffre en route de la faim et s'abandonne au sommeil pour se revigorer. Le premier trajet est paradigmatique à cet égard. Le motif de la marche s'y fait insistant : après une halte sous un figuier, le jeune garçon « se remit en marche pour le château de M. Bourdon ». " Il marcha longtemps, longtemps, se croyant toujours près de la lisière du bois, et enfin il s'aperçut qu'il ne savait où il était et qu'il s'était perdu» (p. 25). En cours de route, divers dangers menacent le voyageur, qui n'hésite pas à puiser dans les trésors de la nature - pour apaiser sa faim par exemple. Or ces déplacements, dont la perception est rendue équivoque par la prégnance du thème déréalisant du sommeil $^{11}$, ont un fonctionnement double. Le long séjour de Gribouille chez M. Bourdon s'achève en effet par un voyage en carrosse (le protagoniste a alors quinze ans) qui prend l'allure d'une initiation, occasion de mettre en place un épisode imaginaire qui se présente comme le redoublement métaphorique du voyage. Gribouille, qui croit tomber dans le creux de l'arbre (le grand chêne) qui en était la destination assignée, explore ainsi bien malgré lui « la capitale des fourmis », après avoir traversé une ruche assiégée « qui était devenue un lac de sang » (p. 59), le tout dans une série d'aventures parallèles. Ce premier épisode met en place un dispositif qui donne tout son sens au voyage dans le conte : le véritable voyage coïncide moins avec les différents déplacements des personnages dans le récit qu' avec ce glissement dans le registre métaphorique, qui révèle en l'occurrence une compartimentation signifiante des espaces, tributaire d'une forte axiologie, à connotations philosophiques et politiques. Sous la houlette de M. Bourdon, Gribouille est précipité dans un univers belliqueux, dénué de tout sentiment humanitaire.

\footnotetext{
${ }^{11}$ Un premier endormissement est à l'origine de l'histoire, puisque c'est pendant un somme que Gribouille fait connaissance pour la première fois de M. Bourdon (voir p. 16-19). Arrivé au château, le protagoniste ignore quel est le degré de fiabilité de sa perception : il « ne savait point au juste s'il était éveillé ou endormi » (p. 27). Plus tard, sous le grand chêne, « tout aussitôt, il se sentit accablé d'un grand sommeil, et il ne lui sembla plus voir ni entendre M. Bourdon que dans un rêve » (p. 55).
} 
C'est d'ailleurs pour cette raison que la séquence est suivie d'une fuite cette fois délibérée de l'enfant, qui tente d'échapper à l'influence funeste de son maître. Gribouille, alors poursuivi par le bourdon redevenu insecte et «se voyant sur le point d'être dévoré », «perdit la tête et se précipita dans le ruisseau dont il descendit le courant à la nage » (p. 64), bientôt guidé par une libellule magique, qui déclenche une forte pluie pour décourager le poursuivant. Le premier volet du conte s'achève ainsi sur une réappropriation du proverbe à l'origine du titre : ce Gribouille crédule et simple d'esprit, que mentionne le «Sermon des fous » de 1548. Ce sont ses frères et sœurs, sous les yeux desquels il dérive, qui prononcent le fameux adage, à peine réadapté, qui sert ainsi de clôture à la première partie : "Fin comme Gribouille, qui se jette dans l'eau par crainte de la pluie » (p. 66). Mais la réappropriation qui en faite est double : d'une part parce que Gribouille inverse le proverbe en sauvant sa vie pour échapper à celui qui l'incite à dominer et à exploiter autrui, d'autre part parce que ce geste lui confère pour la première fois une forme d'autonomie : « il recommença à nager aussi vite que le ruisseau, qui était devenu un torrent et qui roulait aussi vite qu'une flèche » (p. 66).

Le véritable voyage, celui qui donne tout son sens au récit, structure en réalité la seconde partie, qui exploite les ressources du conte merveilleux de façon encore plus manifeste :

Lorsque Gribouille eut fait environ deux cents lieues à la nage, il se sentit un peu fatigué et il eut faim, quoiqu'il eût fait tout ce chemin en moins de deux heures. Il y avait longtemps qu'il ne descendait plus le cours du ruisseau et qu'il naviguait en pleine mer sans s'en apercevoir, car il lui semblait rêver et ne pas bien savoir ce qui se passait autour de lui. Il ne voyait plus la demoiselle bleue ; il est à croire qu'elle l'avait quitté lorsque le ruisseau s'était jeté dans une rivière, laquelle rivière s'était jetée dans un fleuve, lequel fleuve avait conduit Gribouille jusqu'à la mer. (p. 67)

L'ouverture de l'espace à des contrées inconnues, l'amplitude du mouvement libéré de l'autorité parentale (réelle ou de substitution), permettent au personnage de changer de statut. C'est d'ailleurs à la suite de ce passage qu'apparaît l'unique occurrence du substantif « voyage », dans un contexte cependant troublant. Car Gribouille a perdu forme humaine : «Il fut étonné de se voir dans cet état et de reconnaître que son voyage l'avait changé en une branche de chêne qui flottait sur l'eau. » (p. 68) On voit par là que le voyage, dynamique associée au changement de lieu, de paysage et d'identité, a essentiellement valeur de déplacement générique dans ce récit. 


\section{Voyage au pays des genres}

Gribouille, type populaire, est d'emblée transféré dans le domaine du conte, comme le signale l' incipit : «Il y avait une fois un père et une mère » (p.9). Par suite, à l'intérieur du récit, les déplacements du personnage corroborent ce premier ancrage. D'abord parce qu'ils s'effectuent au gré de stations qui sont autant d'occasions pour le merveilleux de s'insinuer dans le texte (par le biais des pauses oniriques et des rencontres surnaturelles), ensuite et surtout parce que le protagoniste évolue dans un univers conjointement spatial et générique. Par exemple, l'arrivée de Gribouille au château de M. Bourdon dans la première partie se traduit par l'affleurement de stylèmes caractéristiques de l'écriture du conte de fées. Ainsi, lors de son séjour il constate, à propos des fêtes fastueuses qui sont données dans ce lieu magique : "Tout cela était $s i$ beau, si beau, que les étrangers en étaient éblouis » (p. 51, je souligne). Dans l'énoncé, la conjonction très caractéristique de la « causalité narrative et [de l'] usage hyperbolique des intensives » fonctionne comme un marqueur qui permet d'identifier l'appartenance générique, comme l'a précisément montré Jean-Michel Adam dans son analyse des contes de Perrault ${ }^{12}$. La tournure, conforme à la majorité des incipits perraltiens, témoigne d'une association des étapes du voyage à l'avancée du lecteur à l'intérieur d'un genre codé.

Or la seconde partie, qui transporte Gribouille dans un autre espace, sur l'île des fleurs, est l'occasion d'un ultime transfert, le plus signifiant, qui concerne tout à la fois le personnage et le lecteur. Au voyage en mer qui marque l'articulation des parties un et deux succède un voyage dans les airs (Gribouille en branche de chêne est enlevé par un aigle) qui permet au jeune garçon d'atterrir sans le vouloir en terre inconnue, sur « une grande île déserte où il n'y avait que des arbres, de l'herbe et des fleurs qui brillaient au soleil » (p. 69), dispositif qui réactive l'un des topoï du récit de voyage : la station insulaire, tout en faisant signe vers l'espace de l'utopie et vers la forme de l'apologue.

Finalement transporté « au beau milieu de l'île » par un coup de vent inopiné, Gribouille se retrouve en effet d'un même mouvement au beau milieu des fleurs, dans un monde inconnu et vivant, où l'on n'a de cesse de lui faire fête. À partir de ce moment le récit, toujours placé sous le signe du merveilleux,

\footnotetext{
${ }^{12}$ Voir sur ce point l'étude de Jean-Michel ADAM, qui montre, dans le corpus des Contes ou histoires du temps passé de Perrault, que «l'énoncé inaugural "Il estoit une fois..." [...] est accompagné de constructions syntaxiques similaires et de répétitions de lexèmes qui tissent entre les incipit de multiples relations de sens. » Ute HEIDMANN et Jean-Michel ADAM, Textualité et intertextualité des contes, Paris, Garnier, 2010, p. 237.
} 
endosse une fonction doublement philosophique et politique. En effet, lorsque Gribouille retrouve forme humaine comme par enchantement, grâce à un simple baiser de la «Reine des prés » (p. 74), sa « marraine » (p. 75) ${ }^{13}$, c'est-àdire lorsqu'il devient — selon la formule titre — «le véritable Gribouille» (p. 75), le récit accède dans le même temps à sa véritable dimension, qui dépasse de loin la portée modeste que l'écrivain prétend lui assigner dans sa dédicace. La formule titre ici partiellement reprise - Histoire du véritable Gribouille doit s'entendre comme le mode de lecture d'un texte plus complexe qu'il ne veut bien le laisser paraître.

Ma chère mignonne, je te présente ce petit conte et souhaite qu'il t'amuse pendant quelques heures de ton heureuse convalescence.

En gribouillant ce Gribouille, j'ai songé à toi. Je ne te l'offre pas pour modèle, puisque, en fait de bon cœur et de bon esprit, c'est toi qui m'en a servi.

Sous couvert d'un conte innocent, destiné à un enfant qui en serait l'inspiratrice, George Sand élabore un récit certes charmant, mais autrement plus sophistiqué. L'histoire de Gribouille telle que retranscrite dans ces pages n'est pas celle que délivre la sagesse populaire. Il ne s'agit pas d'un naïf dont les tribulations font rire. Le « véritable » Gribouille (comme le suggère malgré tout le titre) est à chercher ailleurs : c'est un personnage qui accède à sa propre vérité en découvrant les hommes grâce au voyage, mais un voyage dans le monde des idées, ce qu'indique l'écrivain en promenant son lecteur d'un genre à un autre.

Car le récit tend à mettre en évidence une réflexion sociale et humanitaire. En fait, le personnage arrive à l'île des Fleurs et y séjourne longuement pour mieux en repartir. Le voyageur parvenu à maturité, c'està-dire en l'occurrence apte à conceptualiser, se devra de retourner dans le monde de violence, d'exactions et de souffrance auquel il a échappé par ses tribulations merveilleuses, en un geste sacrificiel qui seul pourra apporter la paix aux hommes. La structure narrative est simple : il est d'abord donné à Gribouille de séjourner pendant cent ans sur l'île enchantée, où se déroule une fête perpétuelle. Pour décrire ce séjour, les topoï merveilleux s'accumulent, tissant des liens continus avec le conte, forme matricielle de ce récit : il n'est évidemment pas fortuit de retrouver, par exemple, une parenthèse de cent ans - qui s'apparente à une forme de latence, de sommeil du héros en voie

${ }^{13}$ Le topos du baiser merveilleux se redouble ici vraisemblablement d'une réécriture des Métamorphoses d'Apulée. Gribouille, tel Lucius au contact d'Isis, redevient lui-même. 
d'éducation -, ou encore la séquence bien connue des fées au berceau : la Reine des prés explique ainsi avoir élu l'enfant pour le « douer de douceur et de bonté, ce qui, à [ses] yeux, était le plus beau présent [qu'elle] puisse [lui] faire. » (p. 84-85) Mais le clivage spatial (l'isolement de l'île) se redouble d'un clivage générique. En effet, est-il dit, Gribouille est « sorti du monde des méchants » (p. 78), ce qui signifie tout à la fois qu'il en est issu et qu'il en est provisoirement sauvé. Or sur cette île des délices qui réinvente l'âge d'or, la magie consiste à apprendre les vertus de l'amour, comme le souligne le discours de la reine des fées :

— Eh bien, dit la reine, que veux-tu donc savoir de plus beau et de plus vrai ? Tu sais ce que les hommes de ton pays ne savent pas, ce qu'ils ont absolument oublié, ce dont ils ne se doutent même plus. Tu es magicien, Gribouille, tu es un bon génie. (p. 94)

Dès lors le conte merveilleux troque son attirail surnaturel au profit d'une réflexion idéologique. C'est dans ce but que George Sand renouvelle le type du magicien : loin de faire des prodiges, il est celui qui distingue le Bien du Mal et met son savoir au service des peuples. Cette modification du personnel du conte va de pair avec un nouveau déplacement générique : le conte de fées s'articule désormais avec un canevas qui est celui de l'apologue.

Or la jonction des deux genres - qui communiquent ici - se fait grâce au thème du voyage, cette fois consciemment choisi. Le jeu constant des toponymes dans le récit illustre ce glissement : dans le conte, on se déplace du « carrefour Bourdon» (p. 13) à la « capitale des fourmis » (p. 59) puis, dans la seconde partie, comme on va le voir, de « la contrée des fleurs » (p. 83) au « royaume des bourdons » (p. 84). Rien de bien notable en apparence, si ce n'est la prégnance du monde naturel et le mode figuré de la représentation. En revanche, l'antithèse entre «l'île des Fleurs » (p. 99) et « Bourdonopolis » (p. 107) est plus parlante. La topographie fait désormais système. La "polis » est en effet la cité politiquement constituée, quand "l'île des Fleurs » est désignation de l'espace de l'utopie, ce que corrobore le texte de 1844, «Les Fleurs de mai ». Cette évolution autorise une lecture rétroactive qui permet de déceler dans l'ensemble des désignations topographiques du récit un signifié de type axiologique, qui en pointe la dimension allégorique, j'y reviendrai. 
S'il est relativement difficile de proposer une définition stable et complète de l'apologue, qui se confond souvent avec la fable ${ }^{14}$, on peut dégager un certain nombre d'invariants qui éclairent le fonctionnement du texte sandien. K. Alfons Knauth fait la proposition suivante :

Un apologue serait donc un récit relativement court, à double sens propre et figuratif, caractérisé par un certain ton plaisant, humoristique ou satirique, ainsi que par une fonction instructive ou morale ${ }^{15}$.

Le dispositif allégorique qui s'avère propre au genre était certes présent dès la première partie du conte et dessinait une sorte de cartographie morale. M. Bourdon explique à Gribouille les vices et les vertus respectifs des abeilles, des bourdons et des fourmis $(H G$, p. 60) et conclut : " Imbécile [...], tes parents sont des frelons qui ont oublié leur origine, mais qui n'en ont pas moins tous les instincts et toutes les habitudes de leur race. » (p. 62). L'espèce animale est donc au service d'une analyse qui permet de définir les hommes par ricochet. Mais c'est à partir du moment où Gribouille décide de rejoindre Bourdonopolis, dans un ultime voyage qui préserve la poésie propre au conte merveilleux - puisqu'il se déplace dans un étrange navire, fait d'une feuille de rose - , que le système allégorique et la surdétermination éthique de la fiction s'imposent. La mission de l'enfant est de convertir les hommes, corrompus et rendus insensibles par l'attrait du gain. Pour ce faire, celui-ci peut avoir recours aux vertus apaisantes des fleurs, dont il emporte avec lui un bouquet ${ }^{16}$. La jonction avec l'apologue s'effectue très précisément avec l'arrivée de Gribouille chez les hommes : manichéisme, caricature, mais aussi traits plaisants servent alors une histoire dont les acteurs sont des mariniers ou des gens du peuple, mais tout aussi bien des animaux — les rats, les araignées ou les lézards qui tiennent compagnie à l'enfant dans son cachot, dotés de parole, mais aussi les frelons, les bourdons et les abeilles, qui combattent «l'armée des oiseaux » dirigée par la Reine des fées. Sand, comme c'est souvent le cas, privilégie le syncrétisme des genres littéraires ; aussi ne choisit-elle pas entre le conte et la fable, dont on retrouve ici les composantes mêlées. Mais c'est

\footnotetext{
${ }^{14}$ Voir sur ce point K. Alfons KNAUTH, « Apologue », Dictionnaire raisonné de la caducité des genres littéraires, Genève, Droz, 2014, Saulo NEIVA et Alain MONTANDON (dir.), p. 57 à 70.

${ }^{15}$ Ibid., p. 59.

${ }^{16}$ La Reine des fées explique à Gribouille, avant son départ : « je te permets [...] de cueillir dans mes prés autant de fleurs que tu en voudras emporter, et chaque fois que tu feras respirer la moindre de ces fleurs à un mortel, tu le verras s'adoucir et devenir plus traitable : c'est à ton esprit de faire le reste. » (p. 96)
} 
très clairement un combat d'idées que mettent en scène les différents acteurs, combat violent qui s'achèvera par la victoire de la concorde. La morale propre à l'apologue est doublement assurée par le discours conclusif de la reine et par une ultime séquence narrative, qui montre des armées de fourmis venir dévorer « les cadavres des insectes morts et mourants » $(H G$, p. 122) En suivant le cheminement, d'abord imposé, puis consciemment choisi, du protagoniste, de lieu en lieu puis de pays en pays, le lecteur voyage ainsi à travers des espaces génériques rendus poreux par de multiples interférences, mais néanmoins parfaitement identifiables. En même temps, la nature du personnage est rendue labile par son statut de voyageur qui lui permet, à son insu, de passer de la sphère du conte à celle de l'apologue sans pour autant s'en trouver dénaturé.

En écrivant ce récit allégorique, Sand s'inscrit dans une tradition, qu'elle personnalise en se la réappropriant. En effet « [les] protagonistes de l'apologue sont stéréotypés. Ce sont surtout des animaux, mais aussi des plantes, des objets ou des phénomènes de la nature ${ }^{17} \gg$. Si l'écrivain ne déroge pas à la règle, elle privilégie l'univers floral, qui pour cette raison constitue une contrée bien spécifique dans la fiction. Bourdonopolis diffère de l'île des Fleurs comme un espace géographique se distingue d'un autre, mais ils s'opposent comme s'opposent des concepts ou des croyances antagonistes ${ }^{18}$. Il n'est d'ailleurs pas indifférent qu'une troisième formulation de la morale prenne la forme des Fables antiques et place l'accent sur le motif floral.

L'apologue intègre en effet un épisode dramatique : Gribouille doit se sacrifier pour permettre la victoire de la Reine des fées sur les armées du roi Bourdon. Mais la fin du personnage n'a rien de radical : l'enfant est l'objet d'une métamorphose, qui rappelle fortement les Fables ovidiennes : s'il ne reste en apparence rien de lui sur le bûcher où son corps s'est consumé, « au faîte de cette montagne, on vit s'épanouir une belle fleur que l'on nomme souvenezvous de moi. La reine des prés cueillit cette fleur et la mit dans son sein » avant d'aller combattre ses ennemis (HG, p. 124). L'avenir de Gribouille « devenu petite fleur bleue » est assuré par un ultime transfert, spatial et générique :

Sa marraine l'emporta dans son île, où, pour tout le reste de l'existence des fées, existence dont personne ne connaît le terme, il fut alternativement pendant cent ans petite fleur bleue, bien tranquille et bien heureuse au bord d'un ruisseau,

\footnotetext{
${ }^{17}$ K. Alfons KNAUTH, op. cit., p. 59.

${ }^{18}$ La séquence où la rose est vaincue par le roi des bourdons est représentative à cet égard : « celuici enfonça son dard empoisonné dans le cœur de la plus belle rose. Un cri perçant et une grosse larme s'échappèrent du sein de la rose, et Gribouille, saisi d'horreur et de désespoir, laissa tomber le bouquet » (p. 108).
} 
dans la prairie enchantée, et pendant cent ans jeune et beau sylphe, dansant, chantant, riant, aimant et faisant fête à sa marraine (p. 125).

Le recours final à la mythologie, dont le mode de signification peut rejoindre celui de l'apologue, permet de redoubler la morale idéaliste de la fiction. C'est que le myosotis participe de l'ensemble de ces végétaux spirituels qui, dans le sillage de la fleur bleue chère à Novalis ${ }^{19}$, brillent à l'horizon des rêves chez l'homme du XIX ${ }^{\mathrm{e}}$ siècle. La fleur, donc, signifie en soi et son message de paix et d'harmonie est redoublé par son nom populaire, issu d'une ancienne légende germanique. On note que la formule - Vergissmein-nicht, Ne m'oublie pas - se transforme ici en son équivalent positif : souvenez-vous de moi, où un destinataire multiple, qui désigne la collectivité, se substitue à un interlocuteur privé. La légende à l'origine de cette appellation, légende d'amour et de mort, devient fable politique, en ces temps troublés où s'annonce le coup d'état de décembre 1851. La façon dont se construit le signifié témoigne d'un fonctionnement qui renouvelle en partie le code floral. Dès lors, il serait abusif de considérer le myosotis comme l'un de ces « froids emblèmes de l'impérissable harmonie ${ }^{20} »$ dont le narrateur de l'article de 1844 redoute l'artifice.

\section{Voyage intertextuel}

Le conte, qui narre les tribulations de Gribouille, se nourrit à l'évidence de formules, de références et de schémas connus. Ainsi, le dispositif initial réécrit l'incipit du Petit Poucet de Charles Perrault, dont il se démarque à peinesinon par une expansion conséquente qui donne notamment plus de consistance aux figures parentales. De part et d'autre sont présentées au lecteur deux familles humbles de sept enfants ${ }^{21}$ dont le plus jeune, le protagoniste éponyme, est marginalisé et devient le souffre-douleur de la fratrie. "Gribouille était le plus petit », écrit Sand (p. 9), c'est-à-dire à la fois le plus jeune et le plus faible, rappelant par là la position de Poucet : «Ce qui les chagrinait encore, c'est que le plus jeune était fort délicat et ne disait mot : prenant pour bêtise ce qui était

\footnotetext{
${ }^{19}$ Dans Heinrich von Ofterdingen, la fleur bleue a forte une dimension spirituelle. Elle représente l'aspiration vers l'absolu, et la possibilité d'une transfiguration du monde par la poésie.

${ }^{20}$ Les Fleurs de mai, op. cit., p. 137.

${ }^{21}$ Ces enfants sont « tous Garçons » chez Perrault (Contes, Paris, Garnier/Flammarion, 1991, p. 290), et hormis Gribouille « trois garçons et trois filles » chez Sand (HG, p. 9).
} 


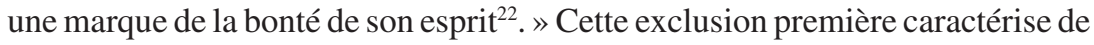
même le héros sandien : «Il n'y avait que le petit Gribouille qui fût maltraité et rebuté, parce qu'il était trop simple et trop poltron, à ce qu'on disait, pour faire comme les autres. » $(H G$, p. 11) Bêtise apparente et bonté profonde font dans les deux cas du personnage voyageur un être au riche potentiel et un sauveur en puissance. Mais, tout en se fondant sur la trame du conte, George Sand en réoriente la moralité. Le Petit Poucet a ceci d'original que le récit propose un double dénouement : le premier insiste sur l'intelligence et le courage du jeune garçon, mais en fait une figure de la mètis, qui toutefois ne recule devant aucun moyen pour enrichir les siens ${ }^{23}$. Sand choisit au contraire d'exploiter le second, à valeur corrective et qui réhabilite pleinement le héros d'un point de vue éthique. Dans cette version, Poucet se met au service du Roi : «il s'en alla à la cour, où il savait qu'on était fort en peine d'une Armée qui était à deux cents lieues de là, et du succès d'une Bataille qu'on avait donnée. ${ }^{24} \gg$ Selon toute vraisemblance, le comportement de Gribouille à Bourdanopolis exploite cet épisode — le plus valorisant pour l'appréhension du personnage — tout en faisant du protagoniste un véritable Héros.

Or en choisissant ce dénouement, l'écrivain permet de substituer une moralité forte à celle, plus pâle, du conte ainsi transformé en apologue. Au gré de son avancée dans le récit, non seulement le lecteur suit les déplacements de Gribouille et, ce faisant, évolue dans un espace générique aux frontières instables, mais il voyage dans une contrée que j'oserai appeler hypotextuelle, d'une référence à une autre. Jamais explicites, celles-ci enrichissent et complexifient la cartographie poétique du récit qu'elles sous-tendent.

Car si l'Histoire du véritable Gribouille prend valeur d'apologue, c'est aussi en raison de ses parentés avec les Fables de La Fontaine. Le recours au monde animal et l'anthropomorphisation des insectes rappelle fortement cet univers, où la morale ouvre ou vient couronner une courte fiction narrative dont les acteurs sont le plus souvent des animaux. Le fait est ici d'autant plus remarquable que George Sand recourt assez rarement à ce procédé, y compris dans les Contes d'une grand-mère qu'elle rédigera à partir de 1872. La fiction présente procède de la rencontre entre Gribouille et un simple bourdon, qui se transformera ensuite en personnage, M. Bourdon gardant sa nature étrangement hybride et réversible tout au long du conte. Époux de la reine des abeilles, il est aussi l'adversaire des cohortes de fourmis qui auront finalement raison de

\footnotetext{
${ }^{22}$ Charles PERRAULT, ibid.

${ }^{23}$ C'est lui qui pousse l'Ogre à égorger ses propres filles, puis qui le dépouille de tous ses biens.

${ }^{24}$ Charles PERRAULT, op. cit., p. 297.
} 
lui. Or les hyménoptères qui constituent pour partie le personnel de la fiction sandienne semblent tout droit issus de la fable «Les frelons et les mouches à miel », qui elle-même reconfigure «Les abeilles et le bourdon jugés par la guêpe » de Phèdre. Si les bourdons disparaissent chez le fabuliste, remplacés par les frelons, le comportement de ces derniers correspond très exactement à la philosophie qu'énonce M. Bourdon dans l'histoire de Gribouille. «Quelques rayons de miel sans maître se trouvèrent, / Des Frelons les réclamèrent », écrit La Fontaine. Et M. Bourdon de déclarer, ayant entrepris de son côté d'expliquer «l'histoire naturelle des frelons et des abeilles »: «Celles-ci travaillent pour leur usage, vous disais-je : elles sont fort habiles, fort actives, fort riches et fort avares. Ceux-là ne travaillent pas si bien et ne savent pas faire le miel ; mais ils ont un grand talent, celui de savoir prendre. » $(H G$, p. 60) L'exposé fait fuir Gribouille qui s'engouffre alors dans une histoire qui lui permettra de construire, en même temps que sa destinée, son propre apologue.

C'est ainsi que le second volet du récit, celui qui consacre véritablement le voyage en posant comme ailleurs idéal l'île des fleurs, transporte le lecteur dans un nouvel univers intertextuel et intericonique, qui donne à la fiction sandienne sa coloration propre. Les fleurs que rencontre l'enfant sont en effet animées, dotées de parole, mais aussi d'une physionomie tout humaine pour certaines d'entre elles. La Reine des prés, « cette belle fleur élégante, menue et embaumée qui vient au printemps et qui aime les endroits frais » $(H G, \mathrm{p}$. 74), finit ainsi par se montrer « sous sa figure naturelle, qui était celle d'une fée plus belle que le jour, plus fraîche que le mois de mai, et plus blanche que la neige ; seulement, elle conservait sa couronne de fleurs de reine des prés, qui, en se mêlant à ses cheveux blonds, semblait plus belle qu' une couronne de perles fines. » (p. 75) Cette fleur-femme est l'objet d'une vignette où Maurice Sand représente en effet une frêle jeune femme, cheveux au vent, le front et le cou cerclés de fleurs, les bras pendant le long de son corps longiligne, revêtu d'une robe étroite. Sa taille ne permet pas de la différencier des graminées qui l'entourent, au bord de l'eau. On ne distingue pas ses pieds : le bas de sa robe reste invisible, perdu dans le fouillis plus sombre des herbes d'où elle surgit. Le mouvement imprimé à la chevelure, la gracilité de la silhouette, presque flexible, font d'elle une fleur parmi les fleurs ${ }^{25}$. L'illustration, plus encore que le texte, évoque Jean-Jacques Grandville ${ }^{26}$, dont Sand connaissait l'œuvre. Dans Les Fleurs animées, préfacé par Alphonse Karr et accompagné de textes

\footnotetext{
${ }^{25}$ La gravure occupe la quasi totalité de la page 74 de notre édition.

${ }^{26}$ Jean Ignace Isidore Gérard (1803-1847), caricaturiste connu sous le nom de Jean-Jacques Grandville dessins zoomorphes que ce dernier est connu, notamment après qu'il a illustré les Fables de Jean de La Fontaine en 1838.
} 
de Taxile Delord ${ }^{27}$, le narrateur annonce une « histoire des Fleurs devenues femmes » (I, 13) que les illustrations de Granville représentent en effet comme autant de figures féminines, que seules différencient leur parure et leur posture, et plongées dans leur milieu naturel dont elles se distinguent à peine. Le jeu de ce probable intertexte permet de préciser le positionnement de Sand dans ce récit faussement anodin. Dans l'ouvrage illustré par Granville en effet, les fleurs révoltées qui tentent de vivre dans le monde des hommes, confrontées à des déceptions ou à des échecs tragiques, choisissent finalement de retourner dans leur pays et de réintégrer leur règne, retour célébré par un bal. Dans l'Histoire du véritable Gribouille, la fête des fleurs célèbre le séjour de l'enfant, éloigné des vicissitudes de l'humanité, et prend l'allure d'un nouvel âge d' ${ }^{28}{ }^{28}$, mais c'est pour mieux valoriser son choix à venir, qui consiste précisément à affronter ce monde pour mieux le convertir.

D'un récit à l'autre, le mouvement s'inverse et le sens de l'apologue se retourne : il ne faut pas oublier que Gribouille est éduqué par une Fée, qui n'est autre que la Reine des fleurs, très consciente de l'état de l'humanité, qu'elle désire améliorer. Chez Delord et Granville, les fleurs retrouvent leur placidité première et le second tome de l'ouvrage en rappelle les fonctions codifiées : composées en bouquets, distillées en parfums, associées à des valeurs fixes, elles restaurent leur fonctionnement premier, emblématique, celui-là même que George Sand fustigeait en 1844, et que le conte de 1850 réfute à nouveau. Dans Les Fleurs animées, ces dernières précisément cessent de l'être dès leur retour ; elles perdent d'ailleurs leur apparence anthropomorphique. La dernière vignette du conte apologue en revanche met en scène l'éternelle reine des prés protégeant le jeune sylphe à la couronne de myosotis, celui dont on ne saurait oublier le pouvoir de conversion et de métamorphose. Cette faculté de changement, qui se traduit par une hybridité essentielle ${ }^{29}$ et pérenne, illustre la richesse du signe et la plurivocité d'un récit que porte et que révèle le voyage qui le structure, par les mutations qui lui sont inhérentes.

L'Histoire du véritable Gribouille est donc celle d'une évolution identitaire à la dimension ouvertement métadiscursive. Si l'enfant charme les

\footnotetext{
${ }^{27}$ L'ouvrage est d'abord publié entre février 1846 et janvier 1847, en 83 livraisons puis, l'année suivante, chez De Gonet en deux volumes, assortis de la Botanique des Dames et l'Horticulture des Dames du comte Foelix. L'ouvrage sera réédité chez Garnier frères en 1867. Les planches, pour cette édition, ont été retouchées par M. Louis Joseph Edouard Maubert, peintre d'histoire naturelle attaché au Jardin des Plantes.

${ }^{28}$ Cf. p. 79 et surtout p. 81 .

${ }^{29}$ La Reine des prés est un végétal et un personnage féminin ; Gribouille est un enfant, une fleur et un être mythologique.
} 
hommes, c'est en partie parce qu'il connaît « une quantité de jolies chansons, fables, contes et apologues que les sylphes lui avaient appris en jouant et en riant dans l'île des Fleurs » $(H G$, p. 104). Il a donc fallu ce transport d'un univers à un autre pour que le langage sorte de la gangue sans doute convenue du conte de fées et déborde vers un plus vaste réseau de significations. En effet Gribouille, armé de son inaltérable bouquet de fleurs, est capable de convertir le langage, dont se déploie la vigueur symbolique. Confiant dans sa mission, l'enfant n'hésite pas à aller au-devant du danger. Dès lors, il se joue des formules apprises :

On s'étonnait de son caractère confiant, et qu'il courût au-devant de tous les dangers ; aussi, sans le connaître pour le véritable Gribouille, lui donna-t-on pour sobriquet son véritable nom : chacun disant qu'il justifiait le proverbe, mais chacun remarquant aussi que le danger semblait le fuir à mesure qu'il s'y jetait. (p. 105)

Comment mieux dire que le héros se joue des clichés, inversant le sens des adages populaires ? Et lorsqu'il meurt en effet, se jetant lui-même sur le bûcher dans un geste sacrificiel, comme on l'a vu, il se métamorphose, choisissant de sauver l'humanité tout en renouvelant le verbe. Voyageur au pays des fleurs, qui disent infiniment plus que ne le suggèrent les manuels d'emblèmes, le véritable Gribouille inscrit ainsi dans la mémoire collective un geste qui est celui-là même du renouveau sémantique et de la mutation des formes. C'est d'abord malgré lui qu'il est parti en quête de sa fleur bleue, hypostase de lui-même comme figure poétique, labile et polysémique : Souvenez-vous de moi... 